\title{
Implantation process of Enterprise IT Application in a medium-sized enterprise
}

\author{
Irving Reascos Paredes \\ Information Systems - Software Engineering \\ North Technical University \\ Ibarra, Ecuador \\ imreascos@utn.edu.ec
}

\author{
João Alvaro Carvalho \\ Centro ALGORITMI \& IS Department \\ University of Minho \\ Guimarães, Portugal \\ jac@dsi.uminho.pt
}

\begin{abstract}
Nowadays, for micro, small, and medium enterprises (SMEs), acquiring generic enterprise IT applications existing in the market, it is more convenient than developing a custom one. Now, the challenge is to search, select, hire, implanting (go-live) and provide continuous maintenance of the enterprise IT application that meets the needs of the enterprise. The problem is the lack of methodologies that cover this process (especially for SMEs), and those that exist are rarely available to the public.

The objective of this article is to identify the process of implantation of an enterprise IT application in medium-sized enterprises. For this, an exploratory case study was carried out in an enterprise that changed the application. In this study, we interview the personnel involved in the implantation process and analyse the documentation generated.
\end{abstract}

The result of this exploratory case study identifies the main motivations, difficulties and lessons learned in the implantation project. Also, an implantation process of an enterprise IT application emerged. This process consists of three phases (pre-implantation, implantation, and post-implantation) and a set of cross-cutting concerns (leadership \& communication, change management, and project management). The phases have 13 stages (pre-implantation - 5, implantation - 6, and post-implantation - 2). This article ends with a discussion of it.

Keywords- Implantation, implementation, enterprise applications, case study, ERP, CRM, SME.

\section{INTRODUCTION}

The hiring of generic enterprise IT application (EITA), sometimes named COTS (Commercial-Off-The-Shelf) or RUSP (Ready to Use Software Product), is nowadays the most common way how enterprises gain access to Information systems. This is especially evident in SMEs, which have limited resources and limited IT capabilities; therefore, they must face more difficulties in dealing with the search, evaluation, selection, hiring, deploys and maintenance of an EITA.

Searching, evaluating, selecting, hiring, deployment and maintenance of EITA, refers to the process that covers all the necessary tasks until a new EITA is embedded - implanted in an enterprise.

The literature reports frequent problems SMEs face when implanting an EITA and the many failures associated this process [1], such as: lack of proper planning [1]; poor leadership [2]; difficulties on the selection of the most adequate EITA [3]; IT capability limitations [4]; high costs of the implantation process [5]; poor fit between the application and the enterprise [6]; poor data quality [7]; user resistance [2]; poor change management [6]; informal communication [3]; difficulties on affording the extensive necessary training [8]; problems with installing the new application in a heterogeneous and often incompatible infrastructure [9]; and difficulties after implantation [1].

The study described in this work is part of a broader research program that aims at developing a set of recommendations to support SME in the process the implanting of an EITA. As an exploratory case study, it aims to contribute to a better understanding of that process.

The study is carried out in an SME that recently substituted an ERP; we consider an ERP as the most common case of EITA. It is not the first time the enterprise faces the implantation of a complex EITA. This refers to a situation that has not been deeply explored, nor is the case for most studies that address enterprises that are implanting an EITA for the first time.

The structure of this article is as follows: Section II describes the enterprise that implanted the EITA, it identifies the main models the implantation (commercial and academic) and justifies the use of the term "implantation." In section III, we present the research design. Section IV shows the implantation process with its different phases, stages, and cross-cutting concerns. Section V presents the difficulties and learned lessons. Finally, section VI shows the discussion of this article, and future work to be done.

\section{BACKGROUND}

\section{A. The car dealer}

The exploratory case study was carried out at a car dealer in northern Ecuador. This enterprise represents an American brand, and therefore, it must abide by the processes and policies imposed by the brand. The enterprise has three main business activities: the sale of vehicles, the sale of spare parts, and car repairs. At the time the study was carried out, the enterprise had around 200 employees in six cities in the north of the country.

This enterprise has more than 30 years of experience in the sale of vehicles. In 2007 they installed their first ERP; on that occasion, the board of directors decided to implant an ERP without consulting with the Information Technology (IT) area. The board informed the IT area that an ERP provider would come to implant the application. 


\section{B. Small and Medium Enterprise (SME)}

Micro, small and medium-sized enterprises (SMEs) play a significant role in the world economy. They are a significant source of entrepreneurial skills, innovation, employment and wealth creation.

Due to their nature, SMEs lack resources and competencies for conducting projects that involve EITA implantation. It is therefore vital to develop methodological support for such projects to be used by SMEs.

\section{EITA implantation models}

In the academic literature, we have found frameworks that guide either some stages of EITA implantation or the entire process in the SME. We have classified the frameworks as follows: decision support [10], [11], preimplantation phase [12]-[15], selection [16], theoretical or conceptual frameworks [5], [17]-[22], cloud adoption [23], and holistic frameworks [24]-[27].

Also, from a professional point of view, the main EITA implantation models are identified: SAP's Activate [28], Microsoft's Dynamics Sure Step [29], Oracle's Unified Method [30], and Primavera's MIP [31]. Most IT consulting enterprises have also developed their own approaches. All these approaches are viewed as corporate assets and are seldom made public; besides, they are focused on larger enterprises.

\section{Implantation vs implementation}

In this article, the process to describe the searching, the evaluation, the selection, the hiring, and the deployment of the EITA will be named as "implantation." This term will be used instead of "implementation," The term "implementation" is ambiguous, and it is used interchangeably to indicate that software will be deployed or developed.

\section{RESEARCH DESIGN}

We conducted this research as an exploratory case study. A Case study research is the most common qualitative method used in information systems field [32]; besides, the case research strategy is well-suited for capturing the knowledge of practitioners and developing theories [33].

The purpose of the case study is to understand how SME performs the searching, evaluation, selection, hiring, deployment, and maintenance of EITA processes. The following are the research questions that drive the study: How is the process of implantation of EITA in SMEs? What are the main difficulties faced by SME when implanting enterprise applications? What are the lessons learned?

Considering the phenomenon of interest, the unit of analysis of this case study consist of a project that involves the implantation of an EITA. The project starts with the identification of business need by the enterprise. It encompasses the search, evaluation, and selection of a commercial software package (EITA), its acquisition, and implantation. The project ends when the enterprise considers that the new EITA works satisfactorily, and it is fully embedded into the enterprise's activities.
To conduct this case study, we used the methodology suggested by Yin [34]. The study can be described as a single case under a rationale of which this case is typical. A holist design was chosen to deal with the unit of analysis: "the project of implantation of an enterprise IT application in SMEs".

To carry out the case study, we obtained an authorisation from the general manager, which was requested through the IT director. Several key players in the implantation project were interviewed, including the technology director, the quality manager, and the implanter of the application - an external enterprise. Later, a second interview with the technology director of the enterprise was necessary to clarify some issues and extending into others. Each interview had an approximate duration of 60 minutes.

The results of these interviews were analysed together with the documents generated during the implantation process; we used MaxQDA qualitative analysis tool following the recommendations of Kuckartz [35] for this purpose. The analysis of the interviews involved the establishment of coding that included categories and subcategories, which arose from the data with an inductive approach. See figure 1.

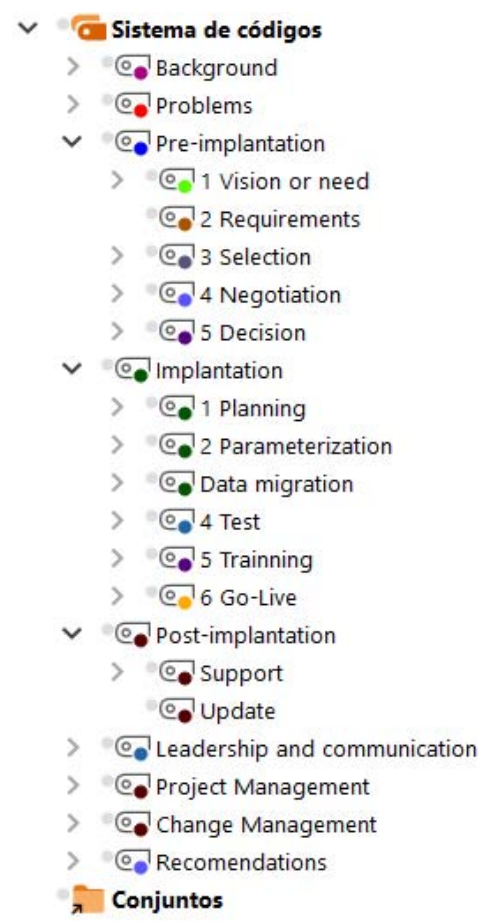

Figure 1: Case study codes using MaxQDA

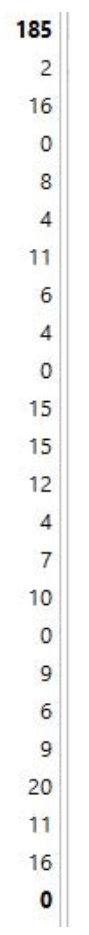

\section{RESUlTS}

Based on the interviews conducted and the documentation reviewed, the implantation process that was carried out in this enterprise emerged, see figure 2. This process consists of three perfectly differentiated phases (preimplantation, implantation, and post-implantation) and a set of cross-cutting concerns (leadership \& communication, change management, and project management). 


\section{A. Pre-implantation phase}

The internal staff of the enterprise conducts the first phase. The objective of this phase is to search, evaluate, select, and contract the new EITA. For which the following stages are carried out:

1) Vision or need: The main reason for the implantation of a new ERP in the enterprise was that government issued a regulation (through the superintendence of banks), which required that enterprises that provide credits should have an EITA that allows to control them.

On the other hand, since some time ago, the enterprise was having difficulties in technical support with the ERP provider. The enterprise was not satisfied with the service received. Also, this service increased in cost. Due to this new need, was made some requests, but in the end, the supplier confirmed that they could not assist in that need specifies.

To solve this problem, the enterprise had two options: 1. Buy a credit module and integrate it with the current ERP; 2. Find another ERP that has all the integrated modules, including the credit module. The vision was to have one or several EITAs that have the information of the enterprise in an integrated way.

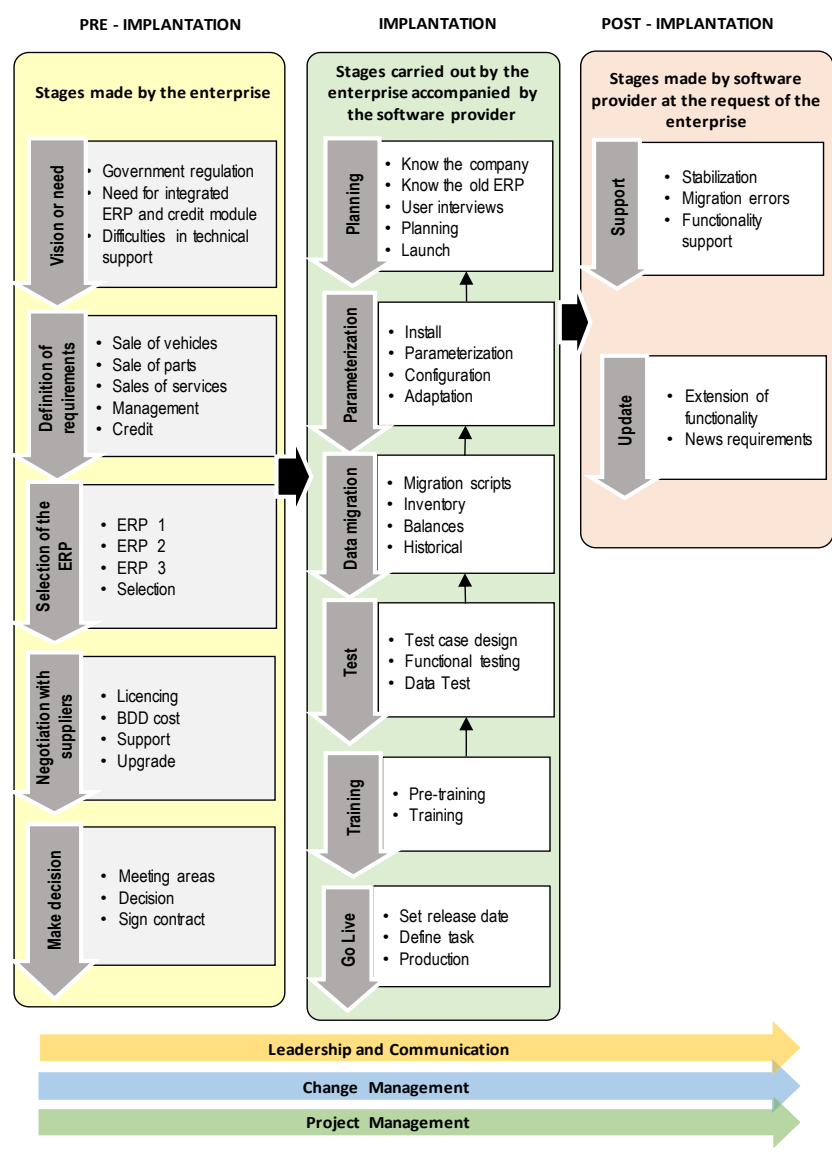

Figure 2: Implantation process of the enterprise IT applications
2) Requirements definition: In general, the EITA that the enterprise was looking should have the following modules, including the credit module requested by the superintendence of banks:

a) Vehicle sales. The sale of vehicles (light, heavy, and used) has some scenarios. The customer can buy a vehicle and add several accessories; then there are several forms of payment: cash, credit, and credit with other financial sources. Depending on that, the enterprise needs to create an amortisation table.

b) Spare parts sales. The vendor can sell a spare; it is not the same as a vehicle bill. It can sell the spare or could assemble kits and sell these kits.

c) Services sales (car workshop). This module requires the client to make reservations for the maintenance of his vehicle. Then, this reservation becomes a workshop order. This module must bill the replaced parts and the services offered.

d) Credit. This module allows managing a customer's credit, registers the credit application, its approval, amortisation table, among others.

e) Management. For the financial and human resources department.

3) ERP Selection: Due to the particularities of this enterprise, it is difficult to find an ERP that fits all areas and is integrated, the company can find suitable software for specific areas, but the drawback is that does not work together, for example, the car workshop has specific issues of its own of the enterprise, it is difficult to find software that meets these requirements and work together with other modules. With these considerations, the enterprise analysed three options:

a) Option 1: Maintain the current ERP and buy a credit module, work on the integration of these systems through web services or other technology. This involves working with two software suppliers to perform integration. The advantage is that the ERP system was in operation, and the external module was sound. The disadvantage is the difficulty of integrating these two applications, the high integration costs and the uncertainty that the apps workintegrated.

b) Option 2: Find another ERP that has the integrated modules, including the credit module. Enterprise staff searched an ERP in the automotive sector; they found another ERP that worked in a concessionaire of characteristics like theirs. The advantages are that the modules are already integrated. The disadvantages were the high cost of this application and that the entire ERP should be migrated.

c) Option 3: Search for a top-level ERP, recognised nationally and internationally that meets the needs of the enterprise. As an advantage of this ERP was the prestige, it possesses as a brand. The disadvantage is the high costs and the uncertainty that it can adapt to this type of requirements. 
Analysing option one among executives, they concluded that the integration would be difficult and possibly must use the two systems independently, for which this option was discarded. Option three was immediately rejected because of its high costs. To analyses the second option, all areas were involved in reviewing the operation of the new ERP. For this review, it was necessary to visit another similar concessionaire to verify how the application worked. Then, a report and presentation were made to management and presidency suggesting this alternative.

It is necessary to indicate that the cost of the second option was higher by $25 \%$ of the first option. Besides, to that the software provider came from a different city $(200 \mathrm{~km})$, implying that the implantation process needed of additional logistics. With this information, the board approved the purchase of a new ERP.

4) Negotiation with the supplier: The mode of licensing of the selected software was per user, and depending on the number of users, the costs varied; the more users the price by user decreased. Also, another cost involved the Database, which almost equalled the value of the ERP itself. This cost at the beginning was not considered due to lack of knowledge of the managers. To solve this inconvenience, the technology director intervened. Other items that were negotiated are the cost of updating and technical support.

5) Make the decision: Initially, the president refused to change the EITA; he said: "They have to adapt to the system that is working, and with that, we will work." The president had information that is transmitted between owners of this type of business. He said that apparently with the same ERP another enterprise was working.

However, the need forces to find a solution. So, based on the second option, a meeting was held between all areas. A joint presentation and report were made to the president and general manager. At this point, the IT director said: "Manager was possibly convinced of the change but needed the criteria of all." In that presentation, the message they conveyed was that the option to change platform was the most convenient. The directors agreed to hold a meeting between the board, and after about a week they said YES.

\section{B. Implantation phase}

Once the provider (the software and its implantation) is hired, it goes on to the next phase, which is carried out by the provider's staff accompanied by the company's technology staff and guided by the provider's implantation methodology. In this phase, the provider identifies the following stages: Planning (knowledge of the enterprise), parameterisation, data migration, testing, training, and the go-live of the EITA.

Also, in the course of the implantation, problems are detected with the technological infrastructure (servers, communication equipment, structured cabling).

1) Planning: The software provider carried out some activities to recognise the current state of the enterprise. So, the provider visited all areas involved with the new ERP, to understand the structure and how the enterprise operates. Interviews were conducted in each area (manager and assistant manager), which are more informed in the daily operation of the enterprise. Another activity is to understand how the old ERP it works and what data structure has.

At this point, the implantation head by the supplier said: "Understanding the strengths and weaknesses of old EITA is essential for the provider."

Besides, the supplier, with the support of the management staff, carries out the constitution, planning and launching the project.

2) Configuration and parameterisation: The ERP provider went on to determine the areas and assemble the data structures by cost centres so that the information delivered by the system is useful. At this stage, meetings are held with key users to gather information; also, with their support, the system is restructured, designed, and coupled with the requirements that the brand (vehicles supplier) imposes.

Once the system is installed, the modules that are needed in the first stage are defined. In this case, the area of sale of vehicles and the financial sector with their respective subsystems are the main ones; others remain for a second stage.

Next, it was the personnel of the enterprise that initiates the input of parameters and data. The following data was entered: agencies, chart of accounts, lines of business, work areas, etc., this with the participation of each manager who knows his area of business. The accountant was included since any inconvenience in any of the areas ends in the accounting area.

Besides, it is necessary to emphasise that there was no need to do a re-engineering or adjustment of the processes. Since the new ERP has the procedures determined by the brand, and the previous one also had these requirements.

3) Migration. For data migration, the provider, together with the technical manager, reviewed the data structure of the old ERP. Subsequently, several scripts were generated to extract data from the previous system; The provider then developed an application in FoxPro to transform and adapt the data to the new database. Finally, it is checked that the information is in the right place.

In theory, migration was smooth, but due to the manager's suggestion, it was decided to migrate the historical data of the previous ERP. This type of data migration was not contemplated in the project, caused additional efforts and lack of information in the new ERP, also delayed the launch of the project three times.

Due to the volume of data, the migration scripts were performed by areas, checks were made on the previous system and the new system, and if something failed, the script was re-performed.

Once the scripts were ready, an attempt was made to migrate the data to an available server in the organisation; but due to the volume and limited equipment, this process took a week and not end. For this reason, it was decided to buy a server that was prepared with seven virtual machines (parallel execution) to perform the migration. With this solution, the final migration took three days. 
In conclusion, migrating historical took three months of additional work. Also, some parts of the system lacked information, which was resolved by entering comments to clarify doubts to users. Due to these factors, the enterprise contracted an external audit to verify that the data is correct.

4) Test. Initial tests were carried out on the enterprise's servers. At this stage, the implantation team determined that the enterprise did not have an adequate physical infrastructure at the level of servers and communications equipment for ERP operation. For this reason, the enterprise improved its technological infrastructure.

The tests were carried out by areas and were closely related to data migration. For example, the implantation team designs the test, then the data is migrated from one system to another, and finally, it is verified that the data match on the two systems. At the same time, the functionality of the EITA is checked.

5) Training: The training was done in conjunction with migration and testing. The provider, together with the technology department migrated information from a specific area. Then met with key personnel in this area, explained the operation of the new EITA, verified data and that EITA is working correctly, this was done cyclically. In this point, the key personnel was already trained, the technology director called it a pre-training.

The final training took place approximately three weeks before passing the ERP to production. To this end, all enterprise personnel involved with the ERP were gathered at the company's headquarters.

6) Go live. Before the ERP went out to production, all areas met to determine the date by which the new system should begin operating. At this meeting, the launch date and the tasks that each area must perform to achieve this objective were determined. Also, at the suggestion of management, it was decided that the previous ERP will work only for consultations.

The output to production was carried out with all the ERP modules at the same time. Since if it is done in phases, it requires much effort from the staff (it involves working with the two ERPs).

According to the software provider: "Putting the new ERP into production in parallel with the old ERP is exhausting, inconvenient and does not give good results."

At this stage, the provider accompanied the enterprise for six months to explain the operation of the ERP, solve some functionality problems, and correct migration errors.

\section{Post-implantation phase}

The third phase is carried out by the software provider in response to a requirement of the enterprise. Also, the costs of this phase are renegotiated every year with the provider. In this phase are the stages of support and update.

1) Support: Initially, the support of the software provider was dedicated to stabilising the system and solving problems that arose in the migration of data. Subsequently, the support focuses on answering concerns about the operation of the system. In support of functionality, there is no inconvenience, i.e., the enterprise requests assistance and the supplier resolve it quickly.

2) Update: The provider assumes the costs of updating the application for three types of contingencies: Tax requirements imposed by the government, requirements imposed by the mark, and any specific needs suggested by the client. In this last option, the provider evaluates if it applies to the enterprises that have the EITA, then the cost is absorbed by the provider. For specific issues of functionality expansion, the contractor must assume these values.

\section{Cross-cutting stages}

This process also identifies transversal stages that are carried out; such as leadership \& communication, project management, and change management.

1) Leadership and communication: The documents show that the manager is the leader of the project, but in practice, the project was led by the technology head and financial head.

A launch event was held for communicating to the employees of this project. In this, all employees were present. The president, the general manager, the software provider, the IT manager, and the process manager talked in this launch. According to the head of IT, "this meeting was crucial for engaging all people."

On the other hand, the ERP provider preferred to conduct face-to-face rather than virtual interviews, according to his words: "it is not the same to see the person on a screen, to be in the enterprise, to feel and understand their processes, and in the function of that to make implantations."

2) Project management: Once the new ERP was selected, and the corresponding contract was signed; a project charter was drawn up between the enterprise and the provider. This document includes the managers of the project by the provider and the enterprise, the description of the project, activities to be carried out, deliverables, milestones, establishes the means of communication, a risk assessment, areas, and human resources participants and finally the times that should take the project.

The implantation of the ERP was planned to be carried out in 6 phases: Project planning, parameterisation, migration, testing, training, and go-live; The scheduled time was 127 days, information that appears in the documents "Certificate of the constitution of the project" and "Plan of management of the project."

However, due to the decision taken by the administration to migrate historical data, the project had a delay of five months. Also, while the system was in operation, they continued to work on historical migration, according to the IT director: "Historical migration took almost a year." According to the provider: "when there is a need to load historical information, demand a lot of effort and time; which the enterprise is not willing to accept. Besides that, the 
principal users cannot allocate the necessary time to the project, due to its multiple occupations."

3) Change management: The enterprise has experience in the implantation of an ERP (which was replaced). Based on this experience, to reduce the resistance to change, it is decided to involve all areas during the search, selection, acquisition, and implantation process of the new ERP.

Subsequently, for the implantation, primary users and secondary users were designated by each one of the existing areas in the enterprise. The primary user was the manager, and the secondary was one of the people who know the whole process of their area. In the meetings that were held, the two users generally attended. For this reason, there was not much resistance to change. Instead, there was collaboration from the people.

On the other hand, with the new ERP, officials reported loss of functionality in some areas. Which is why some claims were justifiable since users were accustomed to having functionality that the new system did not have. However, in general, with the new system, the enterprise gained more functionality and integration than with the previous one module.

When asked if there was resistance to change, the technology director states: "Yes, as in any change. I was also involved in the change of the previous system, and there was also resistance. There will always be resistance. First, the screens are not equal; the application is not the same; the way to call the application is not the same, then if there is a resistance from users. Even users who are not so complicated complain. Also, users who are complicated say the application does not work, and many times, that is not true. It is because they do not know how to use it. "

\section{DifFICULTIES AND LEARNED LESSONS}

\section{A. Difficulties}

- Data migration: The cost and time of data migration were not planned correctly. In the first instance, only initial data was intended to be loaded into the new ERP. However, during the implantation, the manager decided to migrate the historical data, and this delayed the project in approximately six months. Consequently, costs also increased.

- ERP underutilization: There are many things that the system has, but unfortunately, they are not maximising yet.

- Weak technological infrastructure: The existing technological infrastructure was not enough for the new system. For this reason, it was necessary to acquire a new server and update the network of communications.

- Lack of Time: Due to the multiple tasks performed by personnel in the enterprise, it is difficult for people to allocate time to the project.

- Reluctance to change: This is a social issue because many people feel that their enterprise needs a change, but not everybody. For example, Senior managers are convinced that a change needs to be implanted, but their subordinates are not. It is considering that they are the ones who generate information. Besides, in this case, the president of the enterprise (at the beginning) resisted the change to the new ERP.

- Shadow of the previous system: A problem, which the ERP vendor identifies as the shadow of the previous system, "The previous software will always look better than the new software, practically because of the lack of knowledge of the tool in the initial stages, then for about two or three months you have to deal with the shadow of the previous system. This shadow is a difficult subject to jump, Difficult to overcome, although we have more robust tools than the previous".

\section{B. Learned lessons}

- Involve key personnel; for example, the IT director remembers that in the implantation of the previous ERP, there was a financial director who knew in detail the operation of all areas in the enterprise. In the actual implantation, IT director mentions: "In the implantation process, we decided to include the accountant, since any inconvenience found in any of the areas ends in the accounting area, so we decided to include this person there."

- Involve all areas and communicate about the project to reduce the resistance to change. Besides, users are informed of what is being done. In this case, the launch of the project committed people to work on the change of ERP.

- Resistance to change is an inevitable issue; in this case, the president of the enterprise was reluctant to change ERP. To convince him, the staff made a joint presentation in which all the areas presented their points of view.

- The top executives of the enterprise were not aware that the database had an extra cost; which was not part of the ERP negotiation. It was necessary the intervention of the IT director to clarify this issue.

- Ask for a demo to perform functional tests of the application in all areas, since it is not enough to see the presentations. Although it may look attractive and easy to use at first look, people can appreciate the complexity of the system as well as their advantages and disadvantages when using the application.

- Verify that the functionality truly suits what the enterprise needs. In this case, it was not necessary to perform a process re-engineering since the processes existed and were very similar between the two ERPs, which is why only the processes were adapted to the new ERP. In this sense, the software provider mentions that: "the brand manufacturer of the vehicles determines the processes."

- In the negotiation, the functional support requirements must be well established, as well as for the new developments, and even compromise acts that support these extensions of functionality should be signed. 
- Although this enterprise treated the issue of technical support very carefully (due to the negative experience with the previous supplier), there is still a degree of dissatisfaction with the new supplier.

- Deciding to migrate historical data took months of work, delayed the launch of the project up to three times and increased costs. Even after putting into production, it was necessary to continue working on this issue. This decision put the entire project at risk.

- When the enterprise changes its application, some areas may sacrifice functionality, in favour of integrating all areas of the enterprise.

- To obtain a superior performance of the EITA, in parallel with the implantation, an update of the existing technological infrastructure must be carried out.

\section{DISCUSSION}

From this exploratory case study, a process model for the implantation of EITA in medium-sized enterprises has emerged, covering three main phases (pre-implantation, implantation, and post-implantation), Besides cross-cutting concerns (leadership \& communication, change management, and project management). The model provides an overview of the phases, stages, and activities that were carried out in the implantation of an EITA - ERP in a car dealership. The model can also be useful for the enterprises that develop software, resellers, and consultants, as the model identify the stages that an SME goes through before requesting their services.

The difference of this model with the commercial models [28]-[31] is that this model covers the whole process from the perspective of the enterprise. Commercial models carry out this process from the perspective of the supplier and do not necessarily consider the first phase (pre-implementation). Also, if they consider it, they do it to maximize sales.

The difference with the academic models mentioned in section II.B is that this model covers the whole process, from the search, evaluation, selection, hiring, go-live, and continuous maintenance of the EITA.

Academic models focus on solving specific problems, such as support for decision making, selection, preimplantation, among others. There are also theoretical or conceptual models that generally explain the main success factors organized by categories. The holistic models reviewed try to cover the entire process, but they are very general.

A limitation of this model is that it does not provide specific solutions for certain stages. The concern was focused on determining the entire process for the implantation of an EITA (search, evaluation, selection, hiring, deploy, and maintenance).

This exploratory case study served to obtain a general idea of the process of implantation of EITA in SMEs. The work to be developed in the future is to expand the sources of information to software development enterprises, implantation consultants, and SMEs that have recently implanted an EITA. With this information, we hope to understand in depth the process of implementing an EITA.

Also, due to how complicated it was for this enterprise to migrate historical data, in the future, a model could be developed that helps SMEs to request this problem

\section{ACKNOWLEDGEMENT}

The authors acknowledge the support received to produce this work, namely:

i. Universidad Técnica del Norte, Ibarra - Ecuador;

ii. Secretaría Nacional de Educación Superior, Ciencia, Tecnología e Innovación (SENESCYT) - Ecuador;

iii. Project "SmartEGOV: Harnessing EGOV for Smart Governance (Foundations, methods, Tools) / NORTE-010145-FEDER-000037", supported by Norte Portugal Regional Operational Programme (NORTE 2020), under the PORTUGAL 2020 Partnership Agreement, through the European Regional Development Fund (EFDR);

iv. FCT - Fundação para a Ciência e Tecnologia within the Project Scope: UID/CEC/00319/2019 - Portugal;

\section{REFERENCES}

[1] T. H. Nguyen, M. Newby, and M. J. Macaulay, "Information Technology Adoption in Small Business: Confirmation of a Proposed Framework," J. Small Bus. Manag., vol. 53, no. 1, p. 207, Jan. 2015.

[2] L. Shaul and D. Tauber, "CSFs along ERP life-cycle in SMEs: a field study," Ind. Manag. Data Syst., vol. 112, no. 3, pp. 360-384, Mar. 2012.

[3] E. Hustad and D. H. Olsen, "Critical Issues Across the ERP Life Cycle in Small-and-Medium- Sized Enterprises: Experiences from a Multiple Case Study," Procedia Technol., vol. 9, pp. 179-188, 2013.

[4] R. Seethamraju, "Adoption of Software as a Service (SaaS) Enterprise Resource Planning (ERP) Systems in Small and MediumSized Enterprises (SMEs)," Inf. Syst. Front., vol. 17, no. 3, pp. 475492, 2015.

[5] M. I. Nofal and Z. M. Yusof, "Taxonomy framework of ERP success usage in SMEs in middle east region," J. Theor. Appl. Inf. Technol., vol. 86, no. 3, pp. 420-431, 2016.

[6] F. Deltour, "ERP Project in SMEs: A matter of risks, a matter of competencies. A Quantitative analysis," ECIS 2012 Proc., May 2012.

[7] M. J. González, E. Martín, G. Buiza, M. Hidalgo, and J. Beltrán, "Implementation of an operations management system in eight Spanish SMEs," in 2015 International Conference on Industrial Engineering and Systems Management (IESM), 2015, pp. 12961302.

[8] Y.-R. Zeng, L. Wang, and X.-H. Xu, "An integrated model to select an ERP system for Chinese small- and medium-sized enterprise under uncertainty," Technol. Econ. Dev. Econ., vol. 23, no. 1, pp. 38-58, Jan. 2017.

[9] A. Douglas, D. Wainwright, and D. Greenwood, "The Dynamics of IT Supplier Relationships with Construction SMEs: a Technological Approach," UK Acad. Inf. Syst. Conf. Proc. 2010, Mar. 2010.

[10] P. Blackwell, E. M. Shehab, and J. M. Kay, "An effective decisionsupport framework for implementing enterprise IS within SMEs," Int. J. Prod. Res., vol. 44, no. 17, pp. 3533-3552, Sep. 2006.

[11] Y. Xie, C. James Allen, and M. Ali, "An integrated decision support system for ERP implementation in small and medium-sized enterprises," J. Enterp. Inf. Manag., vol. 27, no. 4, pp. 358-384, Jul. 2014.

[12] E. Hustad and D. H. Olsen, "Exploring the ERP pre-implementation process in a small-and-medium-sized enterprise: A case study of a 
Norwegian retail company," presented at the 19th European Conference on Information Systems, ECIS 2011, 2011.

[13] A. N. Hidayanto, M. A. Hasibuan, P. W. Handayani, and Y. G. Sucahyo, "Framework for measuring ERP implementation readiness in small and medium enterprise (SME): A case study in software developer company," J. Comput. Finl., vol. 8, no. 7, pp. 1777-1782, 2013.

[14] I. Jebreen, R. Wellington, and S. G. MacDonell, "Packaged Software Implementation Requirements Engineering by Small Software Enterprises," in 2013 20th Asia-Pacific Software Engineering Conference (APSEC), 2013, vol. 1, pp. 50-57.

[15] S. Ahmadi, C.-H. Yeh, and R. Martin, "Strategic Framework for Achieving Readiness in Organizations to Implement an ERP System," AMCIS 2013 Proc., May 2013.

[16] N. Ganapathy and J. Raju, "A framework for ERP system selection by SME," presented at the 2nd European Conference on Information Management and Evaluation, ECIME, 2008, pp. 193-202.

[17] C. Olupot and G. M. Kituyi, "A Framework for the Adoption of Electronic Customer Relationship Management Information Systems in Developing Countries," Electron. J. Inf. Syst. Dev. Ctries., vol. 58, no. 3, pp. 1-19, Jul. 2013.

[18] H. O. Awa and O. U. Ojiabo, "A model of adoption determinants of ERP within T-O-E framework," Inf. Technol. People, vol. 29, no. 4, pp. 901-930, Nov. 2016.

[19] A. Saedi, "Cloud computing adoption framework: Innovation translation approach," in 2016 3rd International Conference on Computer and Information Sciences (ICCOINS), 2016, pp. 153-157.

[20] S. S. Shahawai and R. Idrus, "Pre-considered factors affecting ERP system adoption in Malaysian SMEs," presented at the UKSim2010 - UKSim 12th International Conference on Computer Modelling and Simulation, 2010, pp. 323-328.

[21] Boumediene Ramdani, Delroy Chevers, and Densil A. Williams, "SMEs' adoption of enterprise applications: A technologyorganisation-environment model," J. Small Bus. Enterp. Dev., vol. 20, no. 4, pp. 735-753, Oct. 2013.

[22] S. Saini, S. Nigam, and S. C. Misra, "Success factors for implementing ERP in SMEs in India: A conceptual model," in 2010 2nd IEEE International Conference on Information Management and Engineering, 2010, pp. 165-169.

[23] S. A. Salim, "Cloud ERP adoption-A process view approach," presented at the Proceedings - Pacific Asia Conference on Information Systems, PACIS 2013, 2013.

[24] R. Jha, M. N. Hoda, and A. K. Saini, "Implementing Best Practices in ERP for Small and Medium Enterprises," in IEEE Symposium on Advanced Management of Information for Globalized Enterprises, 2008. AMIGE 2008, 2008, pp. 1-5.

[25] S. Sahran, F. A. Goni, and M. Mukhtar, "ERP implementation challenges in small and medium enterprise: A framework and case study," Adv. Mater. Res., vol. 139-141, pp. 1636-1639, 2010.

[26] L. X. X. Xu, W. F. Yu, R. Lim, and L. E. Hock, "A methodology for successful implementation of ERP in smaller companies," in Proceedings of 2010 IEEE International Conference on Service Operations and Logistics, and Informatics, 2010, pp. 380-385.

[27] K. E. Fu, "Development of a generic procedure model for the enterprise resource planning implementation in small and medium enterprises," in Proceedings of SICE Annual Conference 2010, 2010, pp. 3523-3528.

[28] "SAP Activate - Speed up your SAP S/4HANA deployment," Lifetime Support by SAP, 2018.

[29] "Microsoft Dynamics Sure Step Methodology Revisited - Microsoft MVP Award Program Blog,” 27-Aug-2012. [Online]. Available: https://blogs.msdn.microsoft.com/mvpawardprogram/2012/08/27/mi crosoft-dynamics-sure-step-methodology-revisited/. [Accessed: 19Apr-2018].
[30] Oracle White Paper, "Oracle Unified Method (OUM) Oracle's Full Lifecycle Method for Deploying Oracle-based Business Solutions." Sep-2016.

[31] Primavera Business Software Solutions, "MIP Projects in motion," 2017. [Online]. Available: http://mip.primaverabss.com/. [Accessed: 27-Apr-2018].

[32] M. D. Myers, "Qualitative Research in Information Systems," MIS Q., vol. 21:2, pp. 241-242, Jun. 1997.

[33] I. Benbasat, D. K. Goldstein, and M. Mead, "The Case Research Strategy in Studies of Information Systems," MIS Q., vol. 11, no. 3, pp. 369-386, 1987.

[34] R. Yin, Case Study Research. Design and Methods, 5th ed. SAGE Publications, 2014.

[35] U. Kuckartz, Qualitative Text Analysis. A guide to methods, practice and using software, First edition. 2014. 